$\mathrm{J}$ o u r n a l of

Mathematics

and Applications

JMA No 41, pp 195-206 (2018)

\title{
An Upper Bound for Third Hankel Determinant of Starlike Functions Related to Shell-like Curves Connected with Fibonacci Numbers
}

\author{
Janusz Sokół, Sedat İlhan and H. Özlem Güney
}

ABstract: We investigate the third Hankel determinant problem for some starlike functions in the open unit disc, that are related to shelllike curves and connected with Fibonacci numbers. For this, firstly, we prove a conjecture, posed in [17], for sharp upper bound of second Hankel determinant. In the sequel, we obtain another sharp coefficient bound which we apply in solving the problem of the third Hankel determinant for these functions.

AMS Subject Classification: 30C45, 30C50.

Keywords and Phrases: Analytic functions; Convex function; Fibonacci numbers; Hankel determinant; Shell-like curve; Starlike function.

\section{Introduction}

Let $\mathcal{A}$ denote the class of functions $f$ which are analytic in the open unit disk $\mathbb{U}=\{z: z \in \mathbb{C}$ and $|z|<1\}$ and let $\mathcal{S}$ denote the class of functions in $\mathcal{A}$ which are univalent in $\mathbb{U}$ and normalized by the conditions $f(0)=f^{\prime}(0)-1=0$ and are of the form:

$$
f(z)=z+\sum_{n=2}^{\infty} a_{n} z^{n} .
$$

We say that $f$ is subordinate to $F$ in $\mathbb{U}$, written as $f \prec F$, if and only if $f(z)=F(w(z))$ for some analytic function $w$ such that $|w(z)| \leq|z|$ for all $z \in \mathbb{U}$. 
If $f \in \mathcal{A}$ and

$$
\frac{z f^{\prime}(z)}{f(z)} \prec p(z) \quad \text { or } \quad 1+\frac{z f^{\prime \prime}(z)}{f^{\prime}(z)} \prec p(z)
$$

where $p(z)=\frac{1+z}{1-z}$, then we say that $f$ is starlike or convex respectively. These functions form known classes denoted by $\mathcal{S}^{*}$ or $\mathcal{C}$ respectively. These classes are very important subclasses of the class $\mathcal{S}$ in geometric function theory. In this paper we consider the following subclass of starlike functions.

Definition 1. The function $f \in \mathcal{A}$ belongs to the class $\mathcal{S} \mathcal{L}$ if it satisfies the condition that

$$
\frac{z f^{\prime}(z)}{f(z)} \prec \tilde{p}(z)
$$

with

$$
\tilde{p}(z)=\frac{1+\tau^{2} z^{2}}{1-\tau z-\tau^{2} z^{2}},
$$

where $\tau=(1-\sqrt{5}) / 2 \approx-0.618$. The class $\mathcal{S} \mathcal{L}$ was introduced in [16].

The function $\tilde{p}$ is not univalent in $\mathbb{U}$, but it is univalent in the disc $|z|<\tau^{2} \approx 0.38$. For example, $\tilde{p}(0)=\tilde{p}(-1 / 2 \tau)=1$ and $\tilde{p}\left(e^{\mp i \arccos (1 / 4)}\right)=\sqrt{5} / 5$, and it may also be noticed that

$$
\frac{1}{|\tau|}=\frac{|\tau|}{1-|\tau|}
$$

which shows that the number $|\tau|$ divides $[0,1]$ such that it fulfils the golden section. The image of the unit circle $|z|=1$ under $\tilde{p}$ is a curve described by the equation given by

$$
(10 x-\sqrt{5}) y^{2}=(\sqrt{5}-2 x)(\sqrt{5} x-1)^{2},
$$

which is translated and revolved trisectrix of Maclaurin. The curve $\tilde{p}\left(r e^{i t}\right)$ is a closed curve without any loops for $0<r \leq r_{0}=(3-\sqrt{5}) / 2 \approx 0.38$. For $r_{0}<r<1$, it has a loop, and for $r=1$, it has a vertical asymptote. Since $\tau$ satisfies the equation $\tau^{2}=1+\tau$, this expression can be used to obtain higher powers $\tau^{n}$ as a linear function of lower powers, which in turn can be decomposed all the way down to a linear combination of $\tau$ and 1 . The resulting recurrence relationships yield Fibonacci numbers $u_{n}$ :

$$
\tau^{n}=u_{n} \tau+u_{n-1} .
$$

In 1976, Noonan and Thomas [10] stated the $s^{\text {th }}$ Hankel determinant for $s \geq 1$ and $k \geq 1$ as

$$
H_{s}(k)=\left|\begin{array}{cccc}
a_{k} & a_{k+1} & \ldots & a_{k+s-1} \\
a_{k+1} & a_{k+2} & \ldots & \vdots \\
\vdots & \vdots & \vdots & \vdots \\
a_{k+s-1} & \ldots & \ldots & a_{k+2(s-1)}
\end{array}\right|
$$

where $a_{1}=1$. 
This determinant has also been considered by several authors. For example, Noor [11] determined the rate of growth of $H_{s}(k)$ as $k \rightarrow \infty$ for functions $f$ given by (1) with bounded boundary. Ehrenborg in [3] studied the Hankel determinant of exponential polynomials. The Hankel transform of an integer sequence and some of its properties were discussed by Layman in [8]. Also, several authors considered the case $s=2$. Especially, $H_{2}(1)=a_{3}-a_{2}^{2}$ is known as Fekete-Szegö functional and this functional is generalized to $a_{3}-\mu a_{2}^{2}$ where $\mu$ is some real number [4]. Estimating for an upper bound of $\left|a_{3}-\mu a_{2}^{2}\right|$ is known as the Fekete-Szegö problem. In [13], Raina and Sokół considered Fekete-Szegö problem for the class $\mathcal{S} \mathcal{L}$. In 1969, Keogh and Merkes [7] solved this problem for the classes $\mathcal{S}^{*}$ and $\mathcal{C}$. The second Hankel determinant is $H_{2}(2)=a_{2} a_{4}-a_{3}^{2}$. Janteng [5] found the sharp upper bound for $\left|H_{2}(2)\right|$ for univalent functions whose derivative has positive real part. Also, in [6] Janteng et al. obtained the bounds for $\left|H_{2}(2)\right|$ for the classes $\mathcal{S}^{*}$ and $\mathcal{C}$. In [17], Sokół et al. considered second Hankel determinant problem for the class $\mathcal{S} \mathcal{L}$ and obtained sharp upper bounds for the functional $\left|a_{2} a_{4}-a_{3}^{2}\right|$ belonging to the class $\mathcal{S} \mathcal{L}$. Also they gave a conjecture for sharp bound of $\left|a_{2} a_{4}-a_{3}^{2}\right|$ for functions in the class $\mathcal{S} \mathcal{L}$. The third Hankel determinant is $H_{3}(1)=a_{3}\left(a_{2} a_{4}-a_{3}^{2}\right)-a_{4}\left(a_{4}-a_{2} a_{3}\right)+a_{5}\left(a_{3}-a_{2}^{2}\right)$. Recently, Babaloa [1], Raza and Malik [15] and Bansal et al. [2] have studied third Hankel determinant $H_{3}(1)$, for various classes of analytic and univalent functions.

In this paper, we investigate an upper bound on the modulus of $H_{3}(1)$ the functions belonging to the class $\mathcal{S} \mathcal{L}$ of analytic functions related to shell-like curves connected with Fibonacci numbers in the open unit disc defined by (1.1).

Now we recall the following lemmas which will be use in proving our main results. Let $\mathcal{P}(\beta), 0 \leq \beta<1$, denote the class of analytic functions $p$ in $\mathbb{U}$ with $p(0)=1$ and $\operatorname{Re}\{p(z)\}>\beta$. Especially, we will use $\mathcal{P}$ instead of $\mathcal{P}(0)$.

Lemma 1.1. ([12]) Let $p \in \mathcal{P}$ with $p(z)=1+c_{1} z+c_{2} z^{2}+\cdots$, then

$$
\left|c_{n}\right| \leq 2, \quad \text { for } \quad n \geq 1 \text {. }
$$

If $\left|c_{1}\right|=2$, then $p(z) \equiv p_{1}(z) \equiv(1+x z) /(1-x z)$ with $x=\frac{c_{1}}{2}$. Conversely, if $p(z) \equiv p_{1}(z)$ for some $|x|=1$, then $c_{1}=2 x$. Furthermore, we have

$$
\left|c_{2}-\frac{c_{1}^{2}}{2}\right| \leq 2-\frac{\left|c_{1}\right|^{2}}{2} .
$$

If $\left|c_{1}\right|<2$, and $\left|c_{2}-\frac{c_{1}^{2}}{2}\right|=2-\frac{\left|c_{1}\right|^{2}}{2}$, then $p(z) \equiv p_{2}(z)$, where

$$
p_{2}(z)=\frac{1+\bar{x} w z+z(w z+x)}{1+\bar{x} w z-z(w z+x)}
$$

and $x=\frac{c_{1}}{2}, w=\frac{2 c_{2}-c_{1}^{2}}{4-\left|c_{1}\right|^{2}}$ and $\left|c_{2}-\frac{c_{1}^{2}}{2}\right|=2-\frac{\left|c_{1}\right|^{2}}{2}$.

Lemma 1.2. ([14]) Let $p \in \mathcal{P}$ with coefficients $c_{n}$ as above, then

$$
\left|c_{1} c_{2}-c_{3}\right| \leq 2
$$


Lemma 1.3. ([9]) Let $p \in \mathcal{P}$ with coefficients $c_{n}$ as above, then

$$
\left|c_{3}-2 c_{1} c_{2}+c_{1}^{3}\right| \leq 2 .
$$

Lemma 1.4. ([16]) If $f(z)=z+\sum_{n=2}^{\infty} a_{n} z^{n}$ belongs to the class $\mathcal{S} \mathcal{L}$, then

$$
\left|a_{n}\right| \leq|\tau|^{n-1} u_{n},
$$

where $u_{n}$ is the sequence of Fibonacci numbers and $\tau=\frac{1-\sqrt{5}}{2}$. Equality holds in (1.8) for the function $f_{0}(z)=\frac{z}{1-\alpha z-\alpha^{2} z^{2}}$.

Lemma 1.5. ([13]) If $f(z)=z+\sum_{n=2} \infty a_{n} z^{n}$ belongs to the class $\mathcal{S L}$, then

$$
\left|a_{3}-\lambda a_{2}^{2}\right| \leq \tau^{2}(2+\lambda) \text { for all } \lambda \in \mathbb{C} \text {. }
$$

The above estimation is sharp. If $\lambda>0$, then the equality in (1.9) is attained by the function $f_{0}(z)=\frac{z}{1-\alpha z-\alpha^{2} z^{2}}$ while by the function $-f_{0}(-z)$, when $\lambda \leq 0$.

Especially, when $\lambda=1$ in (1.9), we obtain $\left|a_{3}-a_{2}^{2}\right| \leq 3 \tau^{2}$.

In this study, we use ideas and techniques used in geometric function theory. The central problem considered here is the sharp upper bounds for the functionals $\left|H_{2}(2)\right|$ and $\left|a_{2} a_{3}-a_{4}\right|$ of functions in the class $\mathcal{S L}$ depicted by the Fibonacci numbers, respectively. Also the third Hankel determinant $\left|H_{3}(1)\right|$ is considered using these functionals.

\section{Main Results}

In [17] it was proved that if $f(z)=z+a_{2} z^{2}+\ldots$ belongs to $\mathcal{S} \mathcal{L}$, then

$$
\left|H_{2}(2)\right|=\left|a_{2} a_{4}-a_{3}^{2}\right| \leq \frac{11}{3} \tau^{4} .
$$

And it was conjectured that $\left|H_{2}(2)\right|=\left|a_{2} a_{4}-a_{3}^{2}\right| \leq \tau^{4}$. Firstly, we present a proof of this.

Theorem 2.1. If $f(z)=z+a_{2} z^{2}+\ldots$ belongs to $\mathcal{S} \mathcal{L}$, then

$$
\left|H_{2}(2)\right|=\left|a_{2} a_{4}-a_{3}^{2}\right| \leq \tau^{4} .
$$

The bound is sharp.

Proof. For given $f \in \mathcal{S} \mathcal{L}$, define $p(z)=1+p_{1} z+p_{2} z^{2}+\cdots$, by

$$
\frac{z f^{\prime}(z)}{f(z)}=p(z)=1+p_{1} z+p_{2} z^{2}+\cdots,
$$


where $p \prec \tilde{p}$. If $p \prec \tilde{p}$, then there exists an analytic function $w$ such that $|w(z)| \leq|z|$ in $\mathbb{U}$ and $p(z)=\tilde{p}(w(z))$. Therefore, the function

$$
h(z)=\frac{1+w(z)}{1-w(z)}=1+c_{1} z+c_{2} z^{2}+\ldots
$$

is in the class $\mathcal{P}$. It follows that

$$
w(z)=\frac{c_{1} z}{2}+\left(c_{2}-\frac{c_{1}^{2}}{2}\right) \frac{z^{2}}{2}+\left(c_{3}-c_{1} c_{2}+\frac{c_{1}^{3}}{4}\right) \frac{z^{3}}{2}+\cdots
$$

and

$$
\begin{aligned}
\tilde{p}(w(z))=1 & +\tilde{p}_{1}\left\{\frac{c_{1} z}{2}+\left(c_{2}-\frac{c_{1}^{2}}{2}\right) \frac{z^{2}}{2}+\left(c_{3}-c_{1} c_{2}+\frac{c_{1}^{3}}{4}\right) \frac{z^{3}}{2}+\cdots\right\} \\
& +\tilde{p}_{2}\left\{\frac{c_{1} z}{2}+\left(c_{2}-\frac{c_{1}^{2}}{2}\right) \frac{z^{2}}{2}+\left(c_{3}-c_{1} c_{2}+\frac{c_{1}^{3}}{4}\right) \frac{z^{3}}{2}+\cdots\right\}^{2} \\
& +\tilde{p}_{3}\left\{\frac{c_{1} z}{2}+\left(c_{2}-\frac{c_{1}^{2}}{2}\right) \frac{z^{2}}{2}+\left(c_{3}-c_{1} c_{2}+\frac{c_{1}^{3}}{4}\right) \frac{z^{3}}{2}+\cdots\right\}^{3}+\cdots \\
=1 & +\frac{\tilde{p}_{1} c_{1} z}{2}+\left\{\left(c_{2}-\frac{c_{1}^{2}}{2}\right) \frac{\tilde{p}_{1}}{2}+\frac{c_{1}^{2}}{4} \tilde{p}_{2}\right\} z^{2} \\
& +\left\{\left(c_{3}-c_{1} c_{2}+\frac{c_{1}^{3}}{4}\right) \frac{\tilde{p}_{1}}{2}+\left(c_{2}-\frac{c_{1}^{2}}{2}\right) \frac{c_{1} \tilde{p}_{2}}{2}+\frac{c_{1}^{3}}{8} \tilde{p}_{3}\right\} z^{3}+\cdots
\end{aligned}
$$

It is known that

$$
\begin{aligned}
\tilde{p}(z) & =1+\sum_{n=1}^{\infty} \tilde{p}_{n} z^{n} \\
& =1+\left(u_{0}+u_{2}\right) \tau z+\left(u_{1}+u_{3}\right) \tau^{2} z^{2}+\sum_{n=3}^{\infty}\left(u_{n-3}+u_{n-2}+u_{n-1}+u_{n}\right) \tau^{n} z^{n} \\
& =1+\tau z+3 \tau^{2} z^{2}+4 \tau^{3} z^{3}+7 \tau^{4} z^{4}+11 \tau^{5} z^{5}+\cdots
\end{aligned}
$$

This shows that the relevant connection of $\tilde{p}$ with the sequence of Fibonacci numbers $u_{n}$, such that $u_{0}=0, u_{1}=1, u_{n+2}=u_{n}+u_{n+1}$ for $n=0,1,2, \ldots$ Thus, $\tilde{p}_{1}=\tau, \tilde{p}_{2}=3 \tau^{2}$ and

$\tilde{p}_{n}=\left(u_{n-1}+u_{n+1}\right) \tau^{n}=\left(u_{n-3}+u_{n-2}+u_{n-1}+u_{n}\right) \tau^{n}=\tau \tilde{p}_{n-1}+\tau^{2} \tilde{p}_{n-2} \quad(n=3,4,5, \ldots)$.

If $p(z)=1+p_{1} z+p_{2} z^{2}+\cdots$, then using (2.4) and (2.5), we have

$$
\begin{gathered}
p_{1}=\frac{c_{1}}{2} \tau \\
p_{2}=\frac{1}{2}\left(c_{2}-\frac{c_{1}^{2}}{2}\right) \tau+\frac{3}{4} c_{1}^{2} \tau^{2},
\end{gathered}
$$


and

$$
p_{3}=\frac{1}{2}\left(c_{3}-c_{1} c_{2}+\frac{c_{1}^{3}}{4}\right) \tau+\frac{3}{2} c_{1}\left(c_{2}-\frac{c_{1}^{2}}{2}\right) \tau^{2}+\frac{1}{2} c_{1}^{3} \tau^{3}
$$

Hence

$\frac{z f^{\prime}(z)}{f(z)}=1+a_{2} z+\left(2 a_{3}-a_{2}^{2}\right) z^{2}+\left(3 a_{4}-3 a_{2} a_{3}+a_{2}^{3}\right) z^{3}+\cdots=1+p_{1} z+p_{2} z^{2}+\cdots$

and

$$
a_{2}=p_{1}, \quad a_{3}=\frac{p_{1}^{2}+p_{2}}{2}, \quad a_{4}=\frac{p_{1}^{3}+3 p_{1} p_{2}+2 p_{3}}{6} .
$$

Therefore, we have

$$
\begin{aligned}
& \left|a_{2} a_{4}-a_{3}^{2}\right| \\
= & \frac{1}{12}\left|p_{1}^{4}-4 p_{1} p_{3}+3 p_{2}^{2}\right| \\
= & \frac{1}{12} \mid \frac{c_{1}^{4}}{16} \tau^{4}-2 c_{1} \tau\left\{\frac{\tau}{2}\left(c_{3}-c_{1} c_{2}+\frac{c_{1}^{3}}{4}\right)+\frac{3 c_{1} \tau^{2}}{2}\left(c_{2}-\frac{c_{1}^{2}}{2}\right)+\frac{c_{1}^{3} \tau^{3}}{2}\right\} \\
& +3\left\{\frac{\tau}{2}\left(c_{2}-\frac{c_{1}^{2}}{2}\right)+\frac{3 c_{1}^{2} \tau^{2}}{4}\right\}^{2} \mid \\
= & \frac{\tau^{2}}{12}\left|\left(\frac{3 c_{1}^{4}}{4}-\frac{3 c_{1}^{2}}{4}\left(c_{2}-\frac{c_{1}^{2}}{2}\right)\right) \tau+\frac{c_{1}^{4}}{2}-c_{1} c_{3}+c_{1}^{2} c_{2}+\frac{3}{4}\left(c_{2}-\frac{c_{1}^{2}}{2}\right)^{2}\right| .
\end{aligned}
$$

It is known (1.2), that

$$
\forall n \in \mathbb{N}, \tau=\frac{\tau^{n}}{u_{n}}-x_{n}, \quad x_{n}=\frac{u_{n-1}}{u_{n}}, \quad \lim _{n \rightarrow \infty} \frac{u_{n-1}}{u_{n}}=|\tau| \approx 0.618
$$

Applying (2.10) gives

$$
\begin{aligned}
\left|a_{2} a_{4}-a_{3}^{2}\right|= & \frac{\tau^{2}}{12} \mid\left(\frac{3 c_{1}^{4}}{4}-\frac{3 c_{1}^{2}}{4}\left(c_{2}-\frac{c_{1}^{2}}{2}\right)\right) \frac{\tau^{n}}{u_{n}}+c_{1}\left(c_{1} c_{2}-c_{3}\right) \\
& +\frac{3}{4} c_{2}\left(c_{2}-\frac{c_{1}^{2}}{2}\right)+\frac{3}{8}\left(2 x_{n}-1\right) c_{1}^{2}\left(c_{2}-\frac{c_{1}^{2}}{2}\right)+\frac{2-3 x_{n}}{4} c_{1}^{4} \mid .
\end{aligned}
$$


Now, applying the triangle inequality, (1.4), (1.5) and (1.6) gives

$$
\begin{aligned}
\left|a_{2} a_{4}-a_{3}^{2}\right| \leq & \frac{\tau^{2}}{12}\left|\frac{3 c_{1}^{4}}{4}-\frac{3 c_{1}^{2}}{4}\left(c_{2}-\frac{c_{1}^{2}}{2}\right)\right| \frac{|\tau|^{n}}{u_{n}} \\
& +\frac{\tau^{2}}{12}\left\{\left|c_{1}\right|\left|c_{1} c_{2}-c_{3}\right|+\frac{3}{4}\left|c_{2}\right|\left|c_{2}-\frac{c_{1}^{2}}{2}\right|\right. \\
& \left.+\frac{3}{8}\left(2 x_{n}-1\right)\left|c_{1}^{2}\right|\left|c_{2}-\frac{c_{1}^{2}}{2}\right|+\frac{2-3 x_{n}}{4}\left|c_{1}\right|^{4}\right\} \\
\leq & \frac{\tau^{2}}{12}\left|\frac{3 c_{1}^{4}}{4}-\frac{3 c_{1}^{2}}{4}\left(c_{2}-\frac{c_{1}^{2}}{2}\right)\right| \frac{|\tau|^{n}}{u_{n}} \\
& +\frac{\tau^{2}}{12}\left\{2\left|c_{1}\right|+\frac{3}{2}\left(2-\frac{\left|c_{1}\right|^{2}}{2}\right)\right. \\
& \left.+\frac{3}{8}\left(2 x_{n}-1\right)\left|c_{1}\right|^{2}\left(2-\frac{\left|c_{1}\right|^{2}}{2}\right)+\frac{2-3 x_{n}}{4}\left|c_{1}\right|^{4}\right\}
\end{aligned}
$$

because by $(2.10)$, we have $x_{n} \rightarrow 0.618$ so $2 x_{n}-1>0,2-3 x_{n}>0$ for sufficiently large $n$. So, in above calculation, in the last line, we have got a function of variable $\left|c_{1}\right|=: y \in[0,2]$ and after elementary calculations we can get that

$$
\max _{y \in[0,2]}\left\{2 y+\frac{3}{2}\left(2-\frac{y^{2}}{2}\right)+\frac{3}{8}\left(2 x_{n}-1\right) y^{2}\left(2-\frac{y^{2}}{2}\right)+\frac{2-3 x_{n}}{4} y^{4}\right\}=12-12 x_{n} \text { at } y=2 \text {. }
$$

Furthermore, it is clear that

$$
\lim _{n \rightarrow \infty}\left|\frac{3 c_{1}^{4}}{4}-\frac{3 c_{1}^{2}}{4}\left(c_{2}-\frac{c_{1}^{2}}{2}\right)\right| \frac{|\tau|^{n}}{u_{n}}=0
$$

and (2.10), (2.11) give

$$
\begin{gathered}
\lim _{n \rightarrow \infty}\left[\max _{y \in[0,2]}\left\{2 y+\frac{3}{2}\left(2-\frac{y^{2}}{2}\right)+\frac{3}{8}\left(2 x_{n}-1\right) y^{2}\left(2-\frac{y^{2}}{2}\right)+\frac{2-3 x_{n}}{4} y^{4}\right\}\right] \\
=12-12|\tau|=12 \tau^{2}
\end{gathered}
$$

so we have

$$
\left|a_{2} a_{4}-a_{3}^{2}\right| \leq 0+\frac{\tau^{2}}{12} 12 \tau^{2}=\tau^{4}
$$

If we take in $(2.2)$

$$
h(z)=\frac{1+z}{1-z}=1+2 z+2 z^{2}+\ldots,
$$

then putting $c_{1}=c_{2}=c_{3}=2$ in (2.9) gives

$$
\left|a_{2} a_{4}-a_{3}^{2}\right|=\frac{\tau^{2}}{12}|12 \tau+12|=\frac{\tau^{2}}{12}\left|12 \tau^{2}\right|=\tau^{4} .
$$

and it shows that (2.1) is sharp. It completes the proof. 
Theorem 2.2. If $f(z)=z+a_{2} z^{2}+\ldots$ belongs to $\mathcal{S} \mathcal{L}$, then

$$
\left|a_{2} a_{3}-a_{4}\right| \leq|\tau|^{3}
$$

The bound is sharp.

Proof. Let $f \in \mathcal{S L}$ and $p \in \mathcal{P}$ where $p(z)=1+p_{1} z+p_{2} z^{2}+\cdots$. From (2.6), (2.7), (2.8) and

$\frac{z f^{\prime}(z)}{f(z)}=1+a_{2} z+\left(2 a_{3}-a_{2}^{2}\right) z^{2}+\left(3 a_{4}-3 a_{2} a_{3}+a_{2}^{3}\right) z^{3}+\cdots=1+p_{1} z+p_{2} z^{2}+\cdots$

we have

$$
a_{2} a_{3}-a_{4}=\frac{1}{3}\left(p_{1}^{3}-p_{3}\right)
$$

So we obtain

$$
\begin{aligned}
& \left|a_{2} a_{3}-a_{4}\right|=\frac{1}{3}\left|p_{1}^{3}-p_{3}\right| \\
= & \frac{1}{3}\left|\frac{c_{1}^{3}}{8} \tau^{3}-\frac{1}{2}\left(c_{3}-c_{1} c_{2}+\frac{c_{1}^{3}}{4}\right) \tau-\frac{3}{2} c_{1}\left(c_{2}-\frac{c_{1}^{2}}{2}\right) \tau^{2}-\frac{1}{2} c_{1}^{3} \tau^{3}\right| \\
= & \frac{1}{3}\left|\left\{\frac{1}{4} c_{1}\left(c_{2}-\frac{c_{1}^{2}}{2}\right)+\frac{1}{2}\left(c_{1} c_{2}-c_{3}\right)-\frac{7}{4} c_{1} c_{2}\right\} \tau+\frac{3}{8} c_{1}^{3}-\frac{3}{2} c_{1} c_{2}\right| .
\end{aligned}
$$

Applying (2.10), we have

$$
\begin{aligned}
& \left|a_{2} a_{3}-a_{4}\right|=\frac{1}{3} \mid\left\{\frac{1}{4} c_{1}\left(c_{2}-\frac{c_{1}^{2}}{2}\right)+\frac{1}{2}\left(c_{1} c_{2}-c_{3}\right)-\frac{7}{4} c_{1} c_{2}\right\} \frac{\tau^{n}}{u_{n}} \\
& -\frac{1}{4} c_{1}\left(c_{2}-\frac{c_{1}^{2}}{2}\right) x_{n}-\frac{1}{2}\left(c_{1} c_{2}-c_{3}\right) x_{n}+\frac{7}{4} c_{1} c_{2} x_{n}+\frac{3}{8} c_{1}^{3}-\frac{3}{2} c_{1} c_{2} \mid . \\
= & \frac{1}{3} \mid\left\{\frac{1}{4} c_{1}\left(c_{2}-\frac{c_{1}^{2}}{2}\right)+\frac{1}{2}\left(c_{1} c_{2}-c_{3}\right)-\frac{7}{4} c_{1} c_{2}\right\} \frac{\tau^{n}}{u_{n}} \\
& +\frac{1}{2}\left(c_{3}-2 c_{1} c_{2}+c_{1}^{3}\right) x_{n}+\frac{3}{4} c_{1}\left(c_{2}-\frac{c_{1}^{2}}{2}\right) x_{n} \\
& +\frac{5}{4} c_{1} c_{2} x_{n}-\frac{3}{4} c_{1}\left(c_{2}-\frac{c_{1}^{2}}{2}\right)-\frac{3}{4} c_{1} c_{2} \mid
\end{aligned}
$$


Now, applying the triangle inequality, (1.4), (1.5),(1.6) and (1.7) gives

$$
\begin{aligned}
& \left|a_{2} a_{3}-a_{4}\right| \leq \frac{1}{3}\left|\left\{\frac{1}{4} c_{1}\left(c_{2}-\frac{c_{1}^{2}}{2}\right)+\frac{1}{2}\left(c_{1} c_{2}-c_{3}\right)-\frac{7}{4} c_{1} c_{2}\right\}\right| \frac{\left|\tau^{n}\right|}{u_{n}} \\
& +\frac{1}{2}\left|c_{3}-2 c_{1} c_{2}+c_{1}^{3}\right| x_{n}+\frac{\left|3 x_{n}-3\right|}{4}\left|c_{1}\right|\left|c_{2}-\frac{c_{1}^{2}}{2}\right|+\frac{\left|5 x_{n}-3\right|}{4}\left|c_{1}\right|\left|c_{2}\right| \\
& \leq \frac{1}{3}\left|\left\{\frac{1}{4} c_{1}\left(c_{2}-\frac{c_{1}^{2}}{2}\right)+\frac{1}{2}\left(c_{1} c_{2}-c_{3}\right)-\frac{7}{4} c_{1} c_{2}\right\}\right| \frac{\left|\tau^{n}\right|}{u_{n}} \\
& +\frac{1}{2}\left|c_{3}-2 c_{1} c_{2}+c_{1}^{3}\right| x_{n}+\frac{\left|3 x_{n}-3\right|}{4}\left|c_{1}\right|\left(2-\frac{\left|c_{1}\right|^{2}}{2}\right)+\frac{\left|5 x_{n}-3\right|}{4}\left|c_{1}\right|\left|c_{2}\right| \\
& \leq \frac{1}{3}\left|\left\{\frac{1}{4} c_{1}\left(c_{2}-\frac{c_{1}^{2}}{2}\right)+\frac{1}{2}\left(c_{1} c_{2}-c_{3}\right)-\frac{7}{4} c_{1} c_{2}\right\}\right| \frac{\left|\tau^{n}\right|}{u_{n}} \\
& +x_{n}+x_{n}\left|c_{1}\right|-\frac{3-3 x_{n}}{8}\left|c_{1}\right|^{3},
\end{aligned}
$$

because by (2.10), we have $x_{n} \rightarrow 0.618$ so $3 x_{n}-3<0,5 x_{n}-3>0$ for sufficiently large $n$. If we put $\left|c_{1}\right|=: y \in[0,2]$ then and after elementary calculations we can get that $h(y)=x_{n}+x_{n} y-\left(3-3 x_{n}\right) y^{3} / 3$ increases in $[0,2]$. Therefore,

$$
\max _{y \in[0,2]}\{h(y)\}=\max _{y \in[0,2]}\left\{x_{n}+x_{n} y-\frac{3-3 x_{n}}{8} y^{3}\right\}=6 x_{n}-3 \text { at } y=2 .
$$

Because

$$
\lim _{n \rightarrow \infty}\left|\frac{1}{4} c_{1}\left(c_{2}-\frac{c_{1}^{2}}{2}\right)+\frac{1}{2}\left(c_{1} c_{2}-c_{3}\right)-\frac{7}{4} c_{1} c_{2}\right| \frac{\left|\tau^{n}\right|}{u_{n}}=0
$$

and by $(2.10)$

$$
\lim _{n \rightarrow \infty}\left[\max _{y \in[0,2]}\left\{x_{n}+x_{n} y-\frac{3-3 x_{n}}{8} y^{3}\right\}\right]=6|\tau|-3=-3(2 \tau+1)=-3 \tau^{3}=3|\tau|^{3},
$$

we have

$$
\left|a_{2} a_{3}-a_{4}\right| \leq 0+\frac{3|\tau|^{3}}{3}=|\tau|^{3} .
$$

If we take in $(2.2)$

$$
h(z)=\frac{1+z}{1-z}=1+2 z+2 z^{2}+\ldots,
$$

then putting $c_{1}=c_{2}=c_{3}=2$ in (2.13) gives

$$
\left|a_{2} a_{3}-a_{4}\right|=|\tau|^{3} .
$$

and it shows that (2.12) is sharp. It completes the proof.

Now, we can obtain an upper bound for $\left|H_{3}(1)\right|$ in the class $\mathcal{S} \mathcal{L}$ as follows:

Theorem 2.3. If $f(z)=z+a_{2} z^{2}+\ldots$ belongs to $\mathcal{S} \mathcal{L}$, then

$$
\left|H_{3}(1)\right| \leq 20 \tau^{6}
$$


Proof. Let $f \in \mathcal{S} \mathcal{L}$. By the definition of third Hankel determinant,

$$
H_{3}(1)=\left|\begin{array}{lll}
a_{1} & a_{2} & a_{3} \\
a_{2} & a_{3} & a_{4} \\
a_{3} & a_{4} & a_{5}
\end{array}\right|=a_{3}\left(a_{2} a_{4}-a_{3}^{2}\right)-a_{4}\left(a_{4}-a_{2} a_{3}\right)+a_{5}\left(a_{3}-a_{2}^{2}\right)
$$

where $a_{1}=1$, we have

$$
\left|H_{3}(1)\right| \leq\left|a_{3}\right|\left|a_{2} a_{4}-a_{3}^{2}\right|+\left|a_{4}\right|\left|a_{4}-a_{2} a_{3}\right|+\left|a_{5}\right|\left|a_{3}-a_{2}^{2}\right| .
$$

Considering Lemma 1.4, Lemma 1.5, Theorem 2.1 and Theorem 2.2 in (2.17), we obtain

$$
\begin{aligned}
\left|H_{3}(1)\right| & \leq\left|a_{3}\right|\left|a_{2} a_{4}-a_{3}^{2}\right|+\left|a_{4}\right|\left|a_{4}-a_{2} a_{3}\right|+\left|a_{5}\right|\left|a_{3}-a_{2}^{2}\right| \\
& \leq 2 \tau^{2} \tau^{4}+3|\tau|^{3}|\tau|^{3}+5 \tau^{2} 3 \tau^{2} \\
& =20 \tau^{6} .
\end{aligned}
$$

\section{Concluding, Remarks and Observations}

In our present article, we have obtained sharp estimates of the third Hankel determinant for the class $\mathcal{S L}$ of analytic functions related to shell-like curves connected with the Fibonacci numbers. Firstly, we have proved a conjecture given in [17] for sharp upper bound of second Hankel determinant. Secondly, we have obtained another sharp coefficient bound which will be used in the problem of finding the upper bound associated with the third Hankel determinant $H_{3}(1)$ for this class. Lastly, we have given an upper bound for functional $\left|H_{3}(1)\right|$ in the class $\mathcal{S} \mathcal{L}$.

\section{References}

[1] K.O. Babalola, On $\mathrm{H}_{3}(1)$ Hankel determinant for some classes of univalent functions, Ineq. Theory Appl. 6 (2007) 1-7.

[2] D. Bansal, S. Maharana, J.K. Prajapat, Third order Hankel determinant for certain univalent functions, J. Korean Math. Soc. 52 (6) (2015) 1139-1148.

[3] R. Ehrenborg, The Hankel determinant of exponential polynomials, Amer. Math. Monthly 107 (2000) 557-560.

[4] M. Fekete, G. Szegö, Eine Bemerkung über ungerade schlichte Funktionen, J. London Math. Soc. 8 (1933) 85-89.

[5] A. Janteng, S. Halim, M. Darus, Coefficient inequality for a function whose derivative has a positive real part, J. Inequal. Pure Appl. Math. 7 (2) (2006) Article 50. 
[6] A. Janteng, S. Halim, M. Darus, Hankel determinant for starlike and convex functions, Int. J. Math. Anal. 1 (13) (2007) 619-625.

[7] F.R. Keogh, E.P. Merkes, A coefficient inequality for certain classes of analytic functions, Proc. Amer. Math. Soc. 20 (1969) 8-12.

[8] J.W. Layman, The Hankel transform and some of its properties, J. Integer Sequences 4 (2001) 1-11.

[9] R.J. Libera, E.J. Złotkiewicz, Coefficient bounds for the inverse of a function with derivative in $\mathcal{P}$, Proc. Amer. Math. Soc. 87 (2) (1983) 251-257.

[10] J.W. Noonan, D.K. Thomas, On the second Hankel determinant of areally mean p-valent functions, Trans. Amer. Math. Soc. 223 (2) (1976) 337-346.

[11] K.I. Noor, Hankel determinant problem for the class of functions with bounded boundary rotation, Rev. Roum. Math. Pures Appl. 28 (8) (1983) 731-739.

[12] Ch. Pommerenke, Univalent Functions, Vandenhoeck und Ruprecht, Göttingen, 1975.

[13] R.K. Raina, J. Sokół, Fekete-Szegö problem for some starlike functions related to shell-like curves, Math. Slovaca 66 (2016) 135-140.

[14] V. Ravichandran, S. Verma, Bound for the fifth coefficient of certain starlike functions, C. R. Acad. Sci. Paris, Ser. I 353 (6) (2015) 505-510.

[15] M. Raza, S.N. Malik, Upper bound of the third Hankel determinant for a class of analytic functions related with Lemniscate of Bernoulli, J. Inequal. Appl. 2013 (2013) Article 412.

[16] J. Sokół, On starlike functions connected with Fibonacci numbers, Folia Scient. Univ. Tech. Resoviensis 175 (1999) 111-116.

[17] J. Sokół, S. İlhan, H.Ö. Güney, Second Hankel determinant problem for several classes of analytic functions related to shell-like curves connected with Fibonacci numbers, TWMS Journal of Applied and Engineering Mathematics 8 (1a) (2018) 220-229.

\section{DOI: $10.7862 / \mathrm{rf} .2018 .14$}

\section{Janusz Sokół}

email: jsokol@ur.edu.pl

ORCID: 0000-0003-1204-2286

Faculty of Mathematics and Natural Sciences

University of Rzeszów 
ul. Prof. Pigonia 1, 35-310 Rzeszów

POLAND

\section{Sedat İlhan}

email: sedati@dicle.edu.tr

ORCID: 0000-0002-6608-8848

Department of Mathematics, Faculty of Science

Dicle University

TR-21280 Diyarbakır

TURKEY

H. Özlem Güney

email: ozlemg@dicle.edu.tr

ORCID: 0000-0002-3010-7795

Department of Mathematics, Faculty of Science

Dicle University

TR-21280 Diyarbakır

TURKEY

Received 08.12.2017

Accepted 11.03.2018 